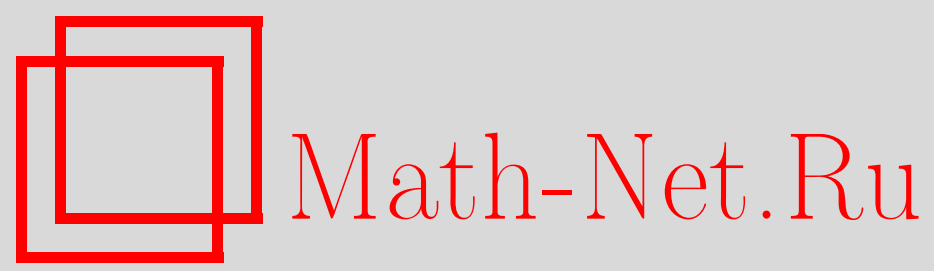

С. Н. Перепечко, Простые выражения для оценки параметра молекулярная свобода в задаче о димерах, Вестник ТвГУ. Серия: Прикладная математика, 2018, выпуск 2, 27-47

DOI: https://doi.org/10.26456/vtpmk194

Использование Общероссийского математического портала Math-Net.Ru подразумевает, что вы прочитали и согласны с пользовательским соглашением

http: //www. mathnet.ru/rus/agreement

Параметры загрузки:

IP: 3.95 .254 .165

26 апреля 2023 г., $17: 27: 23$ 


\title{
МАТЕМАТИЧЕСКАЯ ФИЗИКА
}

УДК $531.19,536.756$

\section{ПРОСТЫЕ ВЫРАЖЕНИЯ ДЛЯ ОЦЕНКИ ПАРАМЕТРА МОЛЕКУЛЯРНАЯ СВОБОДА В ЗАДАЧЕ О ДИМЕРАХ}

\author{
Перепечко С.H.
}

Петрозаводский государственный университет, г. Петрозаводск

Поступила в редакцию 24.10.2017, после переработки 05.02.2018.

Обсуждается метод вычисления параметра молекулярная свобода в модели димеров для двухпараметрических семейств решеточных графов с периодическими граничными условиями. Показано, что схема расчетов для наиболее известных типов решеток может быть унифицирована и позволяет находить простые, но достаточно точные оценки сверху параметра модели. Возможности предложенного подхода проиллюстрированы примерами, для которых ранее были известны только численные значения. Отмечается, что метод может оказаться полезным и для оценки параметров в других решеточных моделях.

Ключевые слова: задача о димерах, молекулярная свобода.

Вестник ТвГУ. Серия: Прикладная математика. 2018. № 2. С. 27-47. https://doi.org/10.26456/vtpmk194

\section{Введение}

В настоящее время задачу о димерах, сформулированную еще в 30-х годах прошлого века, следует рассматривать как одну из классических решеточных моделей статистической механики. В термодинамическом пределе важнейшим параметром этой модели является молекулярная свобода. Однако даже в простейших случаях его вычисление сопряжено со значительными трудностями. Как правило, явные выражения для молекулярной свободы приводят к громоздким интегралам, которые приходится находить численными методами. Те немногочисленные семейства решеточных графов, для которых указанные интегралы могут быть вычислены аналитически, систематизированы в обзоре Ву [1].

Для нахождения молекулярной свободы $\phi$ автором [1] использовался метод Монтролла [2]. В настоящей работе показано, что, в ряде случаев, когда данный метод позволяет получить интегральное представление параметра $\phi$, можно отыскать и более простую оценку сверху, обладающую достаточно высокой точностью. Возможности предложенного подхода проиллюстрированы несколькими примерами из [1], для которых ранее были известны только численные значения.

Рассматриваемый в работе способ построения оценок не ограничивается задачей о димерах. В последнем разделе статьи он применяется для нахождения энтропии остовных деревьев на треугольной решетке. Показано, что использовавшиеся 
ранее методы вычисления этого параметра приводят к интегралам, которые являются частными случаями однопараметрического семейства, допускающего представление в виде явных формул.

\section{1. Сущность метода Монтролла}

C помощью данного метода можно выразить величину $\ln \phi$ в некоторых семействах двухпараметрических решеточных графов в виде двойного интеграла. Находящаяся под знаком интеграла функция является логарифмом от тригонометрического полинома от двух угловых переменных. Явный вид этого полинома зависит от способа построения пфаффовой ориентации и особенностей выполнения процедуры предельного перехода при стремлении параметров графа к бесконечности. Отмеченная особенность приводит к существованию различных эквивалентных выражений для $\ln \phi$.

Практический интерес представляют, естественно, такие выражения, которые позволяют вычислить интеграл по одному из углов в символьном виде. Простейший невырожденный случай соответствует полиному первой степени относительно переменной $\theta$. Тогда, опираясь на соотношение 2.6.36.29 из [3], будем иметь

$$
\int_{0}^{2 \pi} \ln (a+b \cos (\theta)+c \sin (\theta)) d x=2 \pi \ln \left(\frac{a+\sqrt{a^{2}-b^{2}-c^{2}}}{2}\right) .
$$

Часто используемое в расчетах равенство (1) выполняется при $a>0, a^{2}>b^{2}+c^{2}$. С его помощью двойной интеграл сводится к обычному, однако появление радикалов в подынтегральном выражении может существенно усложнить дальнейшие преобразования. Используемый в данной работе подход позволяет строить оценки сверху для интегралов от выражений, фигурирующих в правой части (1), исходя из их тесной связи с обратными гиперболическими функциями.

В основе метода Монтролла, позволяющего находить молекулярную свободу не располагая явными выражениями для числа димерных конфигураций, лежит предположение о том, что матрица пфаффовой ориентации является циркулянтом. С точки зрения структуры графов это предположение соответствует наложению на решетку периодических граничных условий. Сами авторы работ [2] и [1] придерживались распространенной в середине прошлого века точки зрения на то, что трансформация граничных условий не способна изменить величину параметра молекулярная свобода.

Фактически роль граничных условий совершенно нетривиальна (с деталями можно познакомиться в $[4,5])$, поэтому для корректности рассуждений все рассматриваемые в последующих разделах решетки будут считаться уложенными на поверхность тора. В частности, когда пойдет речь о прямоугольных решетках, то будет иметься в виду семейство графов $C_{m} \times C_{n}$, а не $P_{m} \times P_{n}$.

В то же время использованный Монтроллом подход не должен рассматриваться как чересчур частный, поскольку еще в классических работах [6,7] было продемонстрировано совпадение значений $\phi$ в семействах $C_{m} \times C_{n}$ и $P_{m} \times P_{n}$. Экспериментальная проверка аналогичного равенства для треугольных решеток, выполненная в [8] с абсолютной погрешностью, не превышающей $10^{-7}$, также указывает на весьма вероятное совпадение параметров $\phi$ в этих семействах графов. 


\section{2. Прямоугольная решетка}

Этот вид решеток интересен тем, что представляет собой хорошо изученную, разрешимую модель, на которой удобно проиллюстрировать основные особенности используемого в данной работе подхода. Полученное в работах $[6,7]$ значение $\phi$ выражается через постоянную Каталана $G$

$$
\ln \phi=\frac{2 G}{\pi}, \phi=\exp (2 G / \pi) \approx 1,791622812 .
$$

При использовании метода Монтролла можно воспользоваться двойным интегралом из выражения (8) статьи Ву. Однако после применения (1) мы не будем повторять цепочку упрощений из формулы (9) вышеупомянутой работы, а преобразуем полученный результат к виду ${ }^{1}$

$$
\ln \phi=\frac{1}{\pi} \int_{0}^{\pi / 2} \ln (2 \cos (\theta)) d \theta+\frac{1}{\pi} \int_{0}^{\pi / 2} \operatorname{arccosh}\left(\frac{1}{\cos (\theta)}\right) d \theta .
$$

Целесообразность введения обратных гиперболических функций станет более понятной, если воспользоваться еще каким-либо независимым способом вычисления молекулярной свободы. Разрешимость модели позволяет это сделать. На основе явных формул для числа димерных конфигураций в семействе $P_{m} \times P_{n}$ было получено эквивалентное выражение [9]

$$
\ln \phi=\frac{2}{\pi} \int_{0}^{\pi / 2} \operatorname{arcsinh}(\cos (\theta)) d \theta
$$

Если еще учесть, что в соответствии с 2.6.34.8 из справочника [3] первый интеграл (2) обращается в 0, то вполне можно предположить возможность представления $\ln \phi$ в виде интегралов от обратных гиперболических функций достаточно простого вида. В следующих разделах будет показано, что данный прием срабатывает и для других видов решеток, часто использующихся в приложениях.

Особенностью прямоугольных решеток, а точнее следствием разрешимости этой модели оказывается возможность вычисления различных интегралов, возникающих в процессе нахождения параметра $\phi$, с помощью одних и тех же приемов. Так, например, в ходе упрощения (2) на промежутке интегрирования можно воспользоваться равенством $\operatorname{arccosh}(1 / \cos (\theta))=\operatorname{arctanh}(\sin (\theta))$. Тогда, интегрируя почленно степенной ряд для $\operatorname{arctanh}(x)$, получим

$$
\ln \phi=\frac{1}{\pi} \int_{0}^{\pi / 2} \operatorname{arctanh}(\sin (\theta)) d \theta=\frac{1}{\pi} \sum_{k=0}^{\infty} \frac{1}{2 k+1} \int_{0}^{\pi / 2} \sin ^{2 k+1}(\theta) d \theta .
$$

С учетом того, что

$$
\int_{0}^{\pi / 2} \sin ^{2 k+1}(\theta) d \theta=\frac{\sqrt{\pi}}{2} \frac{\Gamma(k+1)}{\Gamma(k+3 / 2)}
$$

\footnotetext{
${ }^{1}$ По причине отсутствия стандартных обозначений названия обратных гиперболических функций взяты такими же, как и в системе компьютерной алгебры Maple.
} 
сумма ряда из предыдущего равенства является значением гипергеометрической функции ${ }_{3} F_{2}$, когда ее аргумент равен 1

$$
\ln \phi=\frac{1}{\pi}{ }_{3} F_{2}\left(\begin{array}{c}
\frac{1}{2}, 1,1 \\
\frac{3}{2}, \frac{3}{2}
\end{array} ; 1\right)
$$

Систематическое появление ${ }_{3} F_{2}$ и других гипергеометрических функций при вычислении $\ln \phi$ в различных семействах решеточных графов позволяет унифицировать с их помощью всю схему проведения расчетов. Разрешимые модели будут характеризоваться такими наборами параметров и аргументов этих функций, которые позволяют воспользоваться известными формулами приведения и выразить полученный результат через известные математические константы.

K прямоугольным решеткам применимо удивительно простое соотношение

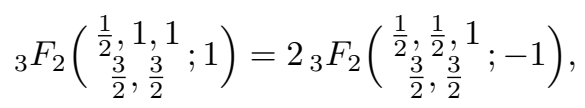

обнаруженное в работе [10]. Тогда, опираясь непосредственно на определение гипергеометрической функции, получаем

$$
\ln \phi=\frac{2}{\pi}{ }_{3} F_{2}\left(\begin{array}{c}
\frac{1}{2}, \frac{1}{2}, 1 \\
\frac{3}{2}, \frac{3}{2}
\end{array} ;-1\right)=\frac{2}{\pi} \sum_{k=0}^{\infty} \frac{(-1)^{k}}{(2 k+1)^{2}}=\frac{2 G}{\pi} .
$$

Попробуем выяснить, существуют ли другие разрешимые модели решеточных графов, в которых молекулярную свободу можно будет вычислить таким же способом, что и в (2). Для этого рассмотрим интеграл более общего вида

$$
I_{1}(\mu)=\int_{0}^{\pi / 2} \operatorname{arccosh}\left(\frac{\mu}{\cos (\theta)}\right) d \theta .
$$

Дифференцируя (3) по $\mu$ в предположении $\mu>1$, находим

$$
I_{1}^{\prime}(\mu)=\int_{0}^{\pi / 2} \frac{d \theta}{\sqrt{\mu^{2}-\cos ^{2}(\theta)}}=\frac{1}{\mu} \mathbf{K}\left(\frac{1}{\mu}\right),
$$

где $\mathbf{K}(x)$ - полный эллиптический интеграл первого рода. Если теперь это соотношение проинтегрировать от 1 до $\mu$, то получим выражение, в котором подынтегральная функция не зависит от $\mu$

$$
I_{1}(\mu)=2 G+\int_{1 / \mu}^{1} \frac{\mathbf{K}(x)}{x} d x .
$$

Поскольку $\mathbf{K}(x)$ является функцией гипергеометрического типа, то и правая часть (4) будет гипергеометрической функцией от $1 / \mu$. Следует, однако, отметить, что в справочной литературе нам не встречались какие-либо особые значения $\mu$, при которых рассматриваемый интеграл удавалось бы представить в более простом виде. Таким образом, в данном классе преобразований разрешимость семейства прямоугольных решеток является уникальным случаем.

Приведение подынтегральных выражений к функции $\operatorname{arccosh}$ в формулах для $\ln \phi$ становится совершенно естественным в контексте получения верхних оценок. 
За основу использованного в работе метода взято легко проверяемое при $z \geqslant 1$ неравенство $\operatorname{arccosh}(z)<\ln (2 z)$. Если аргумент $z$ имеет достаточно простой вид, то интеграл от $\ln (2 z)$ может быть вычислен точно, что и приводит к желаемой оценке. Более того, опираясь на асимптотическое разложение

$$
\operatorname{arccosh}(z)=\ln (2 z)-\sum_{n=1}^{\infty}\left(\begin{array}{c}
2 n \\
n
\end{array}\right) \frac{1}{2 n} \frac{1}{(2 z)^{2 n}}
$$

имеющуюся оценку можно без труда уточнить.

Рассмотрим, к каким результатам приводит изложенный подход в случае прямоугольных решеток. Исходя из соотношения (2), приходим к неравенству

$$
\ln \phi<\frac{1}{\pi} \int_{0}^{\pi / 2} \ln \left(\frac{2}{\cos (\theta)}\right) d \theta=\frac{1}{\pi} \int_{0}^{\pi / 2} \ln (4) d \theta-\frac{1}{\pi} \int_{0}^{\pi / 2} \ln (2 \cos (\theta)) d \theta .
$$

Как уже отмечалось выше, второй интеграл обращается в 0 , поэтому $\phi<2$. Сравнение с точным значением показывает, что погрешность данной оценки составляет примерно $11,6 \%$.

Уточнение полученного результата дает поправку к $\ln \phi$ в виде рациональной дроби

$$
\ln \phi<\ln 2-\frac{1}{\pi} \int_{0}^{\pi / 2}\left(\frac{\cos (\theta)}{2}\right)^{2} d \theta=\ln 2-\frac{1}{16} .
$$

Уточненная оценка превышает $\phi$ менее чем на $4,9 \%$. В данной модели несложно вычислить и вклад $n$-ой поправки, который при больших $n$ оказывается равен $\Theta\left(1 /\left(4 \pi n^{2}\right)\right)$. Медленная сходимость обусловлена упомянутой выше уникальностью семейства прямоугольных решеток. Тем не менее, оценка $\phi<2 \exp (-1 / 16)$, как правило, оказывается вполне практичной.

Для других семейств решеточных графов, являющихся типичными, а не предельными случаями некоторого класса преобразований, наблюдается совершенно иная картина: поправка $n$-го порядка к величине $\ln \phi$ уменьшается со скоростью геометрической прогрессии. Это свойство позволяет получать приближенные формулы для $\phi$, обладающие высокой точностью, не используя процедуры численного интегрирования.

\section{3. Решетка типа 4-8}

Графы этого семейства проще всего представить, рассматривая один из возможных вариантов покрытия плоскости правильными восьмиугольниками и квадратами с одинаковой длиной сторон так, чтобы между четырьмя смежными восьмиугольниками размещался один квадрат. Полагая, что стороны многоугольников образуют набор ребер графа, приходим к изображению решетки, показанному на Рис. 4.5 обзора [2]. Данное название, по-видимому, не является устоявшимся, поскольку в [2] Монтролл использует другой термин - «bathroom-tile lattice».

Как правило, для графов с максимальной степенью вершины 3 характерна сильная зависимость параметра молекулярная свобода от особенностей его строения. Однако в данном случае после наложения периодических граничных условий возникают регулярные двудольные графы степени 3. Для таких графов оценка 
снизу величины $\phi$ хорошо известна [11]. В частности, если степень вершины равна 3 , то $\phi \geqslant 4 / 3$. Посмотрим, насколько истинное значение отличается от указанного.

Выполнив серию преобразований, Ву приходит к интегралу, с помощью которого он численно находит молекулярную свободу

$$
\ln \phi=\frac{1}{2 \pi} \int_{0}^{\pi / 2} \ln \left(\frac{5+\sqrt{25-16 \cos ^{2} \theta}}{2}\right) d \theta, \phi \approx 1,457897968 .
$$

Причина принятого им решения выяснится, если продолжить незавершенные расчеты. Действуя аналогично предыдущему разделу, приведем подынтегральную функцию в (5) к виду, содержащему функцию arccosh

$$
\ln \phi=\frac{1}{2 \pi} \int_{0}^{\pi / 2} \ln (2 \cos (\theta)) d \theta+\frac{1}{2 \pi} \int_{0}^{\pi / 2} \operatorname{arccosh}\left(\frac{5}{4 \cos (\theta)}\right) d \theta .
$$

Из сопоставления (2) и (6) следует, что первый интеграл в (6) обращается в 0, поэтому

$$
\ln \phi=\frac{1}{2 \pi} \int_{0}^{\pi / 2} \operatorname{arccosh}\left(\frac{5}{4 \cos (\theta)}\right) d \theta=\frac{1}{2 \pi} I_{1}\left(\frac{5}{4}\right) .
$$

Полученный результат позволяет говорить о том, что решетки типа 4-8 являются типичным представителем некоторого класса графов, в котором прямоугольные решетки оказываются предельным и, следовательно, разрешимым случаем.

Простейшая оценка сверху находится так же, как и в предыдущем разделе

$$
\ln \phi<\frac{1}{2 \pi} \int_{0}^{\pi / 2} \ln \left(\frac{5}{2 \cos (\theta)}\right) d \theta=\frac{1}{2 \pi} \int_{0}^{\pi / 2} \ln (5) d \theta=\frac{\ln (5)}{4}, \quad \phi<\sqrt[4]{5}
$$

превышая точное значение менее чем на $2,6 \%$. Поскольку $\mu=5 / 4>1$, то можно рассчитывать на более быстрое убывание поправочных членов по сравнению с прямоугольными решетками. Детальный анализ показывает, что в данном случае вклад членов $n$-го порядка составляет $\Theta\left(1 /\left(8 \pi n^{2} \mu^{2 n}\right)\right)$. Ограничиваясь поправками второго порядка, приходим к следующей оценке:

$$
\ln \phi<\frac{\ln (5)}{4}-\frac{1}{50}-\frac{9}{50^{2}}, \quad \phi<\sqrt[4]{5} \exp (-59 / 2500) \approx 1,460 .
$$

\section{4. Шестиугольная решетка}

Двудольные графы, полученные в результате объединения простых циклов $C_{6}$, на протяжении многих лет использовались для изучения свойств ароматических углеводородов. Аналогом димерных конфигураций в таких графах являются структуры Кекуле. Уже к середине прошлого века были найдены аналитические выражения для числа структур Кекуле в нескольких двухпараметрических семействах графов «гексагональных структур» [12].

Асимптотика этих выражений оказалась весьма критичной к тому, какую форму имеет граница решетки. Более того, при фиксации одного из параметров семейства зависимость числа структур Кекуле от второго параметра достаточно часто имеет полиномиальный вид, что эквивалентно значению $\phi=1$. 
В [5] граница решетки задавалась в виде выпуклого симметричного шестиугольника. Благодаря возможности в достаточно широких пределах варьировать длины сторон этого многоугольника, было показано, что молекулярная свобода является функцией границы решетки. Максимальное значение $\phi$ достигалось в случае правильного шестиугольника и оказалось равным $3 \sqrt{3} / 4$. Эта величина меньше нижней оценки $\phi=4 / 3$ для регулярных графов степени 3 , поэтому отказ от планарности посредством перехода к периодическим граничным условиям приведет к значительному увеличению молекулярной свободы.

Применение метода Монтролла позволяет представить $\ln \phi$ в виде двойного интеграла из выражения (13) обзора [1]. Действуя по аналогии с разделом 2, воспользуемся (1) и преобразуем в полученном результате подынтегральное выражение к функции arccosh

$$
\ln \phi=\frac{1}{\pi} \int_{0}^{\pi / 2} \ln (2 \cos (\theta)) d \theta+\frac{1}{\pi} \int_{0}^{\pi / 2} \operatorname{arccosh}\left(\frac{1}{4 \cos (\theta)}+\cos (\theta)\right) d \theta .
$$

С учетом обращения в 0 первого слагаемого (7) вопрос о разрешимости данной модели сводится к изучению двухпараметрического интеграла

$$
I_{2}(\mu, \nu)=\int_{0}^{\pi / 2} \operatorname{arccosh}\left(\frac{\mu}{\cos (\theta)}+\nu \cos (\theta)\right) d \theta
$$

обобщающего $I_{1}(\mu)$. Его детальный анализ крайне сложен, поскольку даже в частном случае $I_{2}(\mu, 0)=I_{1}(\mu)$ результат приводится к достаточно простому виду лишь при $\mu=1$. Однако, благодаря наличию двух параметров, можно построить несколько однопараметрических семейств, допускающих представление (8) в замкнутом виде.

Рассмотрим первое семейство, включающее в себя (7). Для членов этого семейства характерно такое соотношение параметров, при котором логарифмическая форма записи функции arccosh будет иметь аргумент, не содержащий радикалов. Действительно, при всех положительных $x$ справедливо соотношение

$$
\operatorname{arccosh}\left(\frac{1}{2}\left(x+\frac{1}{x}\right)\right)=\left\{\begin{aligned}
\ln (x), & \text { если } x>1 \\
-\ln (x), & \text { если } 0<x \leqslant 1 .
\end{aligned}\right.
$$

Подставляя вместо $x$ величину $2 \cos (\theta) / \mu$, получаем интересующее нас выражение

$$
\operatorname{arccosh}\left(\frac{\mu}{4 \cos (\theta)}+\frac{\cos (\theta)}{\mu}\right)=\left\{\begin{aligned}
\ln (2 \cos (\theta) / \mu), & \text { если } \cos (\theta)>\mu / 2 ; \\
-\ln (2 \cos (\theta) / \mu), & \text { если } 0<\cos (\theta) \leqslant \mu / 2 .
\end{aligned}\right.
$$

При $\mu \geqslant 2$ на всем промежутке интегрирования выполняется второе условие $0 \leqslant \cos (\theta) \leqslant \mu / 2$, поэтому

$$
I_{2}\left(\frac{\mu}{4}, \frac{1}{\mu}\right)=\frac{\pi}{2} \ln (\mu)-\int_{0}^{\pi / 2} \ln (2 \cos (\theta)) d \theta=\frac{\pi}{2} \ln (\mu) .
$$

Нас больше интересует обратная ситуация когда $0<\mu<2$. Тогда, разбивая интеграл на 2 части, приходим к следующему результату

$$
I_{2}\left(\frac{\mu}{4}, \frac{1}{\mu}\right)=\int_{0}^{\arccos (\mu / 2)} \ln \left(\frac{2 \cos (\theta)}{\mu}\right) d \theta-\int_{\arccos (\mu / 2)}^{\pi / 2} \ln \left(\frac{2 \cos (\theta)}{\mu}\right) d \theta .
$$


Полученное выражение можно записать через функцию, которая известна в литературе под названием интеграл Клаузена

$$
\mathrm{Cl}_{2}(\theta)=-\int_{0}^{\theta} \ln \left(2 \sin \left(\frac{t}{2}\right)\right) d t, \quad 0 \leqslant \theta \leqslant \pi .
$$

Опираясь непосредственно на определение этой функции, выражаем фигурирующий в $I_{2}(\mu / 4,1 / \mu)$ интеграл через $\mathrm{Cl}_{2}$

$$
\int_{0}^{\theta} \ln (2 \cos (\phi)) d \phi=\frac{1}{2}\left(\mathrm{Cl}_{2}(\pi-2 \theta)-\mathrm{Cl}_{2}(\pi)\right), \quad 0 \leqslant \theta \leqslant \pi / 2 .
$$

Интеграл Клаузена известен, прежде всего, своим разложением в ряд Фурье

$$
\mathrm{Cl}_{2}(\theta)=\sum_{k=1}^{\infty} \frac{\sin (k \theta)}{k^{2}}, \quad 0 \leqslant \theta \leqslant \pi
$$

из которого сразу же следует, что $\mathrm{Cl}_{2}(0)=\mathrm{Cl}_{2}(\pi)=0$. Это позволяет упростить правую часть предыдущего интеграла. С помощью (9) и очевидного тождества $\arcsin (x)+\arccos (x)=\pi / 2$ получаем окончательный результат

$$
I_{2}\left(\frac{\mu}{4}, \frac{1}{\mu}\right)=\left(2 \arcsin \left(\frac{\mu}{2}\right)-\frac{\pi}{2}\right) \ln (\mu)+\mathrm{Cl}_{2}\left(2 \arcsin \left(\frac{\mu}{2}\right)\right), \quad 0<\mu<2 .
$$

Подставляя $\mu=1$ в (10), находим молекулярную свободу

$$
\ln \phi=\frac{1}{\pi} I_{2}\left(\frac{1}{4}, 1\right)=\frac{1}{\pi} \mathrm{Cl}_{2}\left(\frac{\pi}{3}\right) \approx 0,323065947, \quad \phi=\exp \left(\mathrm{Cl}_{2}(\pi / 3) / \pi\right) .
$$

В [1] Ву отмечает удивительную схожесть интегральных представлений для $\ln \phi$ в шестиугольных и прямоугольньг решетках. Причина этого заключается в том, что в обоих случаях молекулярная свобода выражается через функцию $\mathrm{Cl}_{2}$. Подставляя в ряд Фурье значение $\theta=\pi / 2$, получаем после удаления нулевых членов известный результат $\mathrm{Cl}_{2}(\pi / 2)=G$.

С учетом соотношения 2.6.34.8 из [3] оценка величины $\phi$ сверху приводит к неравенству

$$
\ln \phi<\frac{1}{\pi} \int_{0}^{\pi / 2} \ln \left(\frac{1+4 \cos ^{2}(\theta)}{2 \cos (\theta)}\right) d \theta=\frac{1}{\pi} \int_{0}^{\pi / 2} \ln \left(1+4 \cos ^{2}(\theta)\right) d \theta .
$$

Поскольку интересующий нас интеграл после замены переменных $\theta=\pi / 2-x$ сводится к равенству 2.6.38.1 из [3], то при $b>-1$ получаем

$$
\int_{0}^{\pi / 2} \ln \left(1+b \cos ^{2}(\theta)\right) d \theta=\pi \ln \left(\frac{1+\sqrt{1+b}}{2}\right) .
$$

Выполнив подстановку $b=4$, приходим к «золотому сечению»: $\phi<(1+\sqrt{5}) / 2$. Однако погрешность полученного значения больше чем в двух рассмотренных ранее случаях. Более того, для этой решетки поправочные члены убывают столь 
же медленно, как и на прямоугольной решетке. В результате даже с учетом трех поправок оценка

$$
\phi<\frac{1+\sqrt{5}}{2} \exp \left(-\frac{\sqrt{5}}{25}\left(1+\frac{3}{10}+\frac{52}{375}\right)\right) \approx 1,422667
$$

превышает ф почти на $3 \%$.

$\mathrm{C}$ выражениями, приводящими к функции $\mathrm{Cl}_{2}$, приходится иметь дело и при исследовании других решеточных моделей. В частности, при анализе модели Изинга на треугольной решетке автор [13] столкнулся с величиной идентичной $\ln \phi$ из данного раздела. Не подозревая о соотношении (9), он решил вычислить интеграл численно путем суммирования медленно сходящегося степенного ряда. Однако, несмотря на попытки ускорить сходимость, опираясь на соотношение $2 \mathrm{Cl}_{2}(\pi / 3)=3 \mathrm{Cl}_{2}(2 \pi / 3)$, точность полученного результата оказалась низкой.

Следует отметить, что еще в 1832 году Клаузен составил 16-значные таблицы $\mathrm{Cl}_{2}(\theta)$ с шагом изменения аргумента в $1^{\circ}[14]$. В соответствии с этими таблицами максимальное значение интеграла Клаузена равно $\mathrm{Cl}_{2}\left(60^{\circ}\right)=1,0149416064096536$.

При изучении решеточных моделей функция $\mathrm{Cl}_{2}$ обычно встречается в таком виде, что ее аргумент оказывается рациональным числом, умноженным на $\pi$. В этом случае интеграл Клаузена сводится к производной от $\psi(x)=d / d x \ln (\Gamma(x))$. Наиболее известный результат подобного рода принадлежит Феттису [15]

$$
2 \sqrt{3} \mathrm{Cl}_{2}\left(\frac{\pi}{3}\right)=\psi^{\prime}\left(\frac{1}{3}\right)-\frac{2 \pi^{2}}{3}
$$

Целесообразность повсеместного использования $\psi^{\prime}(x)$ обусловлена способностью многих систем компьютерной алгебры выполнять символьные преобразования над выражениями, содержащими эту функцию.

\section{5. Треугольная решетка}

Изучение этого вида решеток было начато в работе основоположников модели димеров [16]. Однако, прогресс, достигнутый за прошедшее время в изучении данной модели, оказался не столь значительным, как в случае прямоугольных решеток. Несмотря на то, что способ построения пфаффовых ориентаций в указанных графах был описан Монтроллом еще в [2], практичные формулы для числа димерных конфигураций на решетках с периодическими граничными условиями были получены значительно позднее [17]. Аналогичные по структуре формулы для решеток со свободными границами неизвестны. По этой причине общепринятое утверждение о независимости молекулярной свободы от вида граничных условий в данной модели следует рассматривать как гипотезу, хотя и проверенную с высокой точностью [8].

Ранние попытки выразить $\ln \phi$ в явном виде приводили к громоздким интегралам (см., например, работу [18]), не допускающим применение (1). В пятом разделе обзора [1] обсуждаются более компактные формулы. Тем не менее, несмотря на сведение двойного интеграла к обычному, никаких замкнутых выражений для $\phi$ к настоящему времени получить не удалось. 
В работе [1] Ву не поясняет причины невозможности отыскать $\phi$ в явном виде для некоторых типов решеток, однако систематическое применение изложенного в предыдущих разделах метода позволяет выдвинуть ряд предположений. Взяв за основу двойной интеграл из формулы (30) в [1], применим соотношение (1) и преобразуем полученный результат к виду, содержащему функцию arcosh. C учетом четности $\cos (\theta)$ после перехода к половинному углу получаем

$$
\ln \phi=\frac{1}{\pi} \int_{0}^{\pi / 2} \ln (2 \cos (\theta)) d \theta+\frac{1}{\pi} \int_{0}^{\pi / 2} \operatorname{arccosh}\left(\frac{1}{\cos (\theta)}+\cos (\theta)\right) d \theta
$$

Как уже отмечалось ранее, первый интеграл равен 0 , поэтому $\ln \phi=I_{2}(1,1) / \pi$. Таким образом, проблема нахождения явной формулы для молекулярной свободы на треугольной решетке оказывается тесно связанной с поиском дополнительных случаев интегрируемости $I_{2}(\mu, \nu)$ в замкнутом виде.

Если явные выражения для $I_{2}(\mu, \nu)$ имеют достаточно простой вид, то можно полагать, что и соответствующие производные будут описываться столь же простыми формулами. Посмотрим, что собой представляет производная по $\mu$

$$
\frac{\partial}{\partial \mu} I_{2}(\mu, \nu)=\int_{0}^{\pi / 2} \frac{d \theta}{\sqrt{\nu^{2} \cos ^{4}(\theta)+(2 \mu \nu-1) \cos ^{2}(\theta)+\mu^{2}}} .
$$

В общем случае правая часть (14) опять приводит к полным эллиптическим интегралам, так как при $|a|<1$ известно следующее соотношение:

$$
\int_{0}^{\pi / 2} \frac{d \theta}{\sqrt{\cos ^{4}(\theta)+2 a \cos ^{2}(\theta)+1}}=\frac{\mathbf{K}\left(\frac{1}{2} \sqrt{2-\sqrt{2+2 a}}\right)}{\sqrt[4]{2+2 a}} .
$$

Сопоставление с более простой ситуацией, рассмотренной в разделе 2 , не позволяет рассчитывать на возможность ее интегрирования в достаточно общем виде.

Нетрудно заметить, что семейство интегралов $I_{2}(\mu / 4,1 / \mu)$, обсуждавшееся в предыдущем разделе, соответствует такому условию, при котором подкоренное выражение в (14) обращается в полный квадрат. Канонические формы эллиптических интегралов принято записывать через функцию $\sin$. Выполнив такое преобразование над знаменателем правой части (14)

$$
\sqrt{\nu^{2} \sin ^{4}(\theta)-\left(2 \nu \mu+2 \nu^{2}-1\right) \sin ^{2}(\theta)+(\mu+\nu)^{2}-1},
$$

можно получить еще одно интегрируемое семейство.

Обращение в 0 свободного члена тригонометрического полинома при $(\mu+\nu)^{2}=1$ позволяет понизить его степень путем вынесения $\sin (\theta)$ из-под знака корня и, тем самым, избавиться от эллиптических интегралов. В свою очередь, для существования $I_{2}(\mu, \nu)$ требуется выполнение условия $\mu+\nu \geqslant 1$, поэтому из двух вариантов остается только один. Однако интегралы $I_{2}(\mu, 1-\mu)$ непригодны для вычисления молекулярной свободы на треугольных решетках.

Помимо уже упомянутьх выше однопараметрических семейств существуют изолированные наборы параметров $\mu$ и $\nu$, при которых величина $I_{2}(\mu, \nu)$ может быть найдена в замкнутом виде. Один из примеров такого рода будет приведен в 
конце раздела. Однако систематический поиск всех изолированных наборов требует проведения специального исследования, выходящего за рамки данной работы.

Трудности, возникающие при вычислении молекулярной свободы даже в простейших семействах решеточных графов, ставят на повестку дня вопрос о разработке альтернативных численно-аналитических методов нахождения этого параметра, аналогичных, например, [8]. Это особенно актуально для решеток со свободными границами, к которым метод Монтролла неприменим. С другой стороны, возможность получения простой, но достаточно точной оценки, без привлечения процедуры численного интегрирования может оказаться полезной на практике. Такую оценку несложно получить для всех интегралов $I_{2}(\mu, \nu)$.

В рассматриваемом нами случае имеем

$$
\ln \phi<\frac{1}{\pi} \int_{0}^{\pi / 2} \ln \left(\frac{2\left(1+\cos ^{2}(\theta)\right)}{\cos (\theta)}\right) d \theta=\frac{1}{\pi} \int_{0}^{\pi / 2} \ln \left(4\left(1+\cos ^{2}(\theta)\right)\right) d \theta
$$

где уже учтено, что $\int_{0}^{\pi / 2} \ln (2 \cos (\theta)) d \theta=0$. Подставляя значение $b=1$ в (12), получаем $\phi<1+\sqrt{2}$. Превышение этой оценки над точным значением составляет менее $2,5 \%$.

Для нахождения поправочных членов перепишем $\ln \phi$ в виде ряда

$$
\ln \phi=\ln (1+\sqrt{2})-\frac{1}{2 \pi} \sum_{n=1}^{\infty}\left(\begin{array}{c}
2 n \\
n
\end{array}\right) \frac{1}{n 2^{2 n}} \int_{0}^{\pi / 2} \frac{\cos ^{2 n}(\theta) d \theta}{\left(1+\cos ^{2}(\theta)\right)^{2 n}} .
$$

Несмотря на то, что в результате интегрирования возникает гипергеометрическая функция Гаусса

$$
\ln \phi=\ln (1+\sqrt{2})-\frac{1}{2 \sqrt{\pi}} \sum_{n=1}^{\infty}\left(\begin{array}{c}
2 n \\
n
\end{array}\right) \frac{1}{2 n 2^{2 n}} \frac{\Gamma\left(n+\frac{1}{2}\right)}{\Gamma(n+1)}{ }_{2} F_{1}\left(\begin{array}{c}
2 n, n+\frac{1}{2} \\
n+1
\end{array} ;-1\right),
$$

все члены ряда оказываются рациональными числами, поскольку

$$
{ }_{2} F_{1}\left(\begin{array}{c}
2 n, n+\frac{1}{2} \\
n+1
\end{array} ;-1\right)=2_{2}^{\frac{1}{2}-2 n} F_{1}\left(\begin{array}{c}
\frac{1}{2}, 1-n \\
n+1
\end{array} ;-1\right) .
$$

Применяя формулу удвоения для гамма-функции, можно заменить все ее вхождения на факториалы и привести окончательный результат к виду

$$
\ln \phi=\ln (1+\sqrt{2})-\frac{1}{2 \sqrt{2}} \sum_{n=1}^{\infty}\left(\begin{array}{c}
2 n \\
n
\end{array}\right)^{2} \frac{1}{n 2^{6 n}{ }_{2} F_{1}}\left(\begin{array}{c}
\frac{1}{2}, 1-n \\
n+1
\end{array} ;-1\right) .
$$

Заменив бесконечную сумму четырьмя первыми слагаемыми, приходим к оценке

$$
\phi<(1+\sqrt{2}) \exp \left(-\frac{\sqrt{2}}{4}\left(\frac{1}{16}+\frac{21}{4096}+\frac{515}{786432}+\frac{27125}{268435456}\right)\right) \approx 2,356545,
$$

которая отличается от указанного в [1] значения в шестой значащей цифре.

Фигурирующие в (15) гипергеометрические функции удобно находить с помощью рекуррентного соотношения. Если для краткости ввести обозначение $a_{n}={ }_{2} F_{1}(\underset{n+1}{\stackrel{1}{2}, 1-n} ;-1)$, то

$$
4 n(2 n+1)^{2} a_{n+1}=(n+1)\left(32 n^{2}-16 n+5\right) a_{n}-16 n\left(n^{2}-1\right) a_{n-1} .
$$


Это соотношение должно быть дополнено начальными условиями: $a_{1}=1, a_{2}=\frac{7}{6}$.

Высокая точность оценки $\phi$ на основе (15) достигается за счет быстрого убывания членов ряда. Для количественного описания этой закономерности нам потребуется одна вспомогательная лемма

Лемма 1. При $n \rightarrow \infty$ имеет место асимптотика

$$
{ }_{2} F_{1}\left(\begin{array}{c}
\frac{1}{2}, 1-n \\
n+1
\end{array} ;-1\right) \sim \Gamma\left(\frac{1}{4}\right) \frac{\sqrt[4]{n}}{2 \sqrt{\pi}}+O\left(\frac{1}{\sqrt[4]{n}}\right)
$$

Доказательство. Опираясь на формулу Эйлера для гипергеометрической функции, находим интегральное представление

$$
{ }_{2} F_{1}\left(\begin{array}{c}
\frac{1}{2}, 1-n \\
n+1
\end{array} ;-1\right)=\frac{\Gamma(n+1)}{\Gamma\left(\frac{1}{2}\right) \Gamma\left(n+\frac{1}{2}\right)} \int_{0}^{1} \frac{(1-t)^{n-1 / 2} d t}{\sqrt{t}(1+t)^{1-n}}=\frac{\Gamma(n+1)}{\Gamma\left(\frac{1}{2}\right) \Gamma\left(n+\frac{1}{2}\right)} \int_{0}^{1} \sqrt{\frac{1-t}{t}}\left(1-t^{2}\right)^{n-1} d t .
$$

Замена переменных $x=t \sqrt{n}$ позволяет преобразовать последний интеграл к виду

$$
\int_{0}^{1} \sqrt{\frac{1-t}{t}}\left(1-t^{2}\right)^{n-1} d t=\int_{0}^{\sqrt{n}} \frac{\sqrt{1-\frac{x}{\sqrt{n}}}\left(1-\frac{x^{2}}{n}\right)^{n-1}}{\sqrt{x} \sqrt[4]{n}} d x .
$$

Целесообразность выполненной замены обусловлена тем, что

$$
\lim _{n \rightarrow \infty}\left(1-\frac{x^{2}}{n}\right)^{n-1}=\exp \left(-x^{2}\right)
$$

В таком случае для вычисления интеграла с заявленной в формулировке точностью можно ограничиться (16) и двумя первыми членами разложения корня в степенной ряд: $\sqrt{1-x / \sqrt{n}}=1-x /(2 \sqrt{n})+O(1 / n)$. Изменение верхнего предела интегрирования на $\infty$ приводит к поправкам экспоненциально малого порядка, поэтому при $n \rightarrow \infty$

$$
\int_{0}^{1} \sqrt{\frac{1-t}{t}}\left(1-t^{2}\right)^{n-1} d t \sim \int_{0}^{\infty} \frac{\left(1-\frac{x}{2 \sqrt{n}}\right) e^{-x^{2}}}{\sqrt{x} \sqrt[4]{n}} d x=\frac{\Gamma\left(\frac{1}{4}\right)}{2 \sqrt[4]{n}}-\frac{\pi \sqrt{2}}{4 \Gamma\left(\frac{1}{4}\right) n^{3 / 4}}+O\left(\frac{1}{n^{5 / 4}}\right) .
$$

Асимптотика отношения гамма-функций в интегральном представлении ${ }_{2} F_{1}$ находится с помощью формулы Стирлинга

$$
\frac{\Gamma(n+1)}{\Gamma\left(\frac{1}{2}\right) \Gamma\left(n+\frac{1}{2}\right)} \sim \sqrt{\frac{n}{\pi}}+\frac{1}{8 \sqrt{\pi n}}+O\left(\frac{1}{n^{3 / 2}}\right) .
$$

Перемножая полученные выражения, приходим к искомой асимптотике

$$
{ }_{2} F_{1}\left(\begin{array}{c}
\frac{1}{2}, 1-n \\
n+1
\end{array} ;-1\right) \sim \frac{\Gamma\left(\frac{1}{4}\right) \sqrt[4]{n}}{2 \sqrt{\pi}}-\frac{\sqrt{2 \pi}}{4 \Gamma\left(\frac{1}{4}\right) \sqrt[4]{n}}+O\left(\frac{1}{n^{3 / 4}}\right)
$$


Результат леммы 1 свидетельствует о том, что гипергеометрический множитель не оказывает существенного влияния на асимптотику членов ряда (15). Действительно, при больших $n$

$$
\left(\begin{array}{c}
2 n \\
n
\end{array}\right)^{2} \frac{1}{n 2^{6 n}} \sim \frac{1}{\pi n^{2} 4^{n}}+O\left(\frac{1}{n^{3} 4^{n}}\right)
$$

поэтому члены этого ряда убывают быстрее геометрической прогрессии с показателем $1 / 4$.

Сопоставление деталей вычисления $\phi$ в нескольких видах планарньх графов указывает на то, что по отношению к семейству $I_{2}(\mu, \nu)$ между треугольными и шестиугольными решетками существует такая же аналогия, как и между решетками типа 4-8 и прямоугольными по отношению к более простому семейству $I_{1}(\mu)$. Треугольные решетки следует рассматривать как типичного представителя некоторого класса графов, в котором шестиугольные решетки являются предельным и, как следствие, разрешимым в замкнутом виде частным случаем. Для типичных случаев в рамках данного подхода характерны более точные оценки молекулярной свободы, поскольку поправочные члены убывают в геометрической прогрессии.

В завершение обсудим коротко возможность нахождения изолированных наборов $\mu$ и $\nu$, при которых $I_{2}(\mu, \nu)$ может быть выражен в замкнутом виде через гипергеометрические функции. Для иллюстрации рассмотрим однопараметрическое семейство $I_{2}(1, \mu)$. Оно интересно тем, что при $\mu=1$ возникает интеграл, через который выражается молекулярная свобода на треугольной решетке. В свою очередь, в предыдущем разделе было вычислено значение $I_{2}(1,1 / 4)=\pi \ln 2$. Попытаемся выяснить, существуют ли другие значения $\mu$, позволяющие получить явное выражение для $I_{2}(1, \mu)$.

Для этого продифференцируем $I_{2}(1, \mu)$ по $\mu$

$$
I_{2}^{\prime}(1, \mu)=\int_{0}^{\pi / 2} \frac{\cos ^{2}(\theta) d \theta}{\sqrt{\mu^{2} \cos ^{4}(\theta)+(2 \mu-1) \cos ^{2}(\theta)+1}}
$$

и разложим производную в ряд по полиномам Лежандра $P_{k}(z)$

$$
I_{2}^{\prime}(1, \mu)=\frac{\sqrt{\pi}}{2} \sum_{k=0}^{\infty} \frac{(-1)^{k} P_{k}\left(1-\frac{1}{2 \mu}\right) \mu^{k} \Gamma\left(k+\frac{3}{2}\right)}{\Gamma(k+2)} .
$$

Из последнего равенства следует, что можно всегда вывести замкнутое выражение для $I_{2}^{\prime}(1, \mu)$, а, следовательно, и для $I_{2}(1, \mu)$, если при фиксированном $z$ известна зависимость $P_{k}(z)$ от $k$. Такие формулы приводятся в литературе, когда аргумент $z=0, \pm 1$. Случай $z=1$ неинтересен, поскольку он соответствует значению $\mu=\infty$. Когда $z=-1$, то $\mu=1 / 4$ и мы приходим к рассмотренной ранее ситуации. Оставшийся вариант $z=0$ дает новое значение $\mu=1 / 2$.

Опуская громоздкие преобразования, выполненные в системе компьютерной алгебры, выпишем окончательный результат

$$
I_{2}\left(1, \frac{1}{2}\right)=\pi \ln 2+\frac{\pi}{16}{ }_{4} F_{3}\left(\begin{array}{c}
\frac{1}{2}, \frac{1}{2}, \frac{3}{4}, \frac{5}{4} \\
1, \frac{3}{2}, \frac{3}{2}
\end{array} ;-\frac{1}{4}\right)+\frac{3 \pi}{512}{ }_{4} F_{3}\left(\begin{array}{c}
1,1, \frac{5}{4}, \frac{7}{4} \\
2,2,2
\end{array} ;-\frac{1}{4}\right) .
$$

Возможность сколько-нибудь существенного упрощения данного выражения остается открытой, поскольку аргумент гипергеометрической функции отличен от 1. 


\section{6. Остовные деревья на треугольной решетке}

При изучении других решеточньх моделей также приходится сталкиваться с величинами, которые имеют смысл аналогичный параметру $\phi$ в модели димеров. Вполне естественно, что методы их вычисления оказываются весьма похожи на методы нахождения молекулярной свободы, поэтому изложенный в данной работе подход может быть применен для оценки параметров в родственных моделях.

Проиллюстрируем такую возможность на примере расчета энтропии остовных деревьев на треугольной решетке. Тесная связь между остовными деревьями и димерными конфигурациями хорошо известна $[19,20]$. Однако при вычислении энтропии конечный результат может быть выражен в замкнутой форме, в то время как аналогичное выражение для $\phi$ неизвестно.

В 5 разделе обзора [1] Ву отмечает значительное внешнее сходство интегралов в формулах (30) и (31), не указывая, впрочем, причин, по которым только один из них может быть найден в явном виде. Метод вычисления энтропии на основе (31) изложен в [21]. При выполнении расчетов авторы [21] опирались на возможность факторизации подынтегрального выражения, а также использовали ряд искусственных приемов, поэтому возможность обобщения полученного ими результата осталась невыясненной.

Если при вычислении двойного интеграла (31) после применения (1), аналогично предыдущим разделам, привести подынтегральное выражение к виду, содержащему функцию arccosh, то энтропия $S_{t}$ представляется следующим образом:

$$
S_{t}=\frac{2}{\pi} \int_{0}^{\pi / 2} \ln (2 \cos (\theta)) d \theta+\frac{2}{\pi} \int_{0}^{\pi / 2} \operatorname{arccosh}\left(\frac{2}{\cos (\theta)}-\cos (\theta)\right) d \theta .
$$

Как уже отмечалось ранее, первый интеграл обращается в 0, поэтому мы опять можем выразить результат через $I_{2}(\mu, \nu): S_{t}=2 / \pi I_{2}(2,-1)$. Интересующую нас величину удобно рассматривать как частный случай однопараметрического семейства $I_{2}(1+\mu,-\mu)$ при $\mu=1$. Но в таком случае выполняется условие $\mu+\nu=1$ и, в соответствии с выводами предыдущего раздела, все интегралы этого семейства могут быть вычислены в явном виде.

После дифференцирования $I_{2}(1+\mu,-\mu)$ по $\mu$ и приведения стоящего под знаком интеграла выражения к функции $\sin (\theta)$, получаем

$$
I_{2}^{\prime}(1+\mu,-\mu)=\int_{0}^{\pi / 2} \frac{\sin (\theta) d \theta}{\sqrt{\mu^{2} \sin ^{2}(\theta)+2 \mu+1}}=\frac{1}{\mu} \arcsin \left(\frac{\mu}{1+\mu}\right) .
$$

С учетом того, что $I_{2}(1,0)=I_{1}(1)=2 G$, проинтегрируем данное равенство на промежутке от 0 до $\mu$

$$
I_{2}(1+\mu,-\mu)=2 G+\int_{0}^{\mu} \frac{1}{x} \arcsin \left(\frac{x}{1+x}\right) d x=2 G+\int_{0}^{\frac{\mu}{1+\mu}} \frac{\arcsin (x)}{x(1-x)} d x .
$$

Представление последнего интеграла в виде суммы позволяет воспользоваться со- 
отношением 1.7.3.13 из [3], которое будет выполняться при $\mu>0$

$$
\begin{gathered}
I_{2}(1+\mu,-\mu)=2 G+\int_{0}^{\frac{\mu}{1+\mu}} \frac{\arcsin (x)}{x} d x+\int_{0}^{\frac{\mu}{1+\mu}} \frac{\arcsin (x)}{1-x} d x= \\
2 G+\arcsin \left(\frac{\mu}{1+\mu}\right) \ln \left(\frac{2 \mu}{1+\mu}\right)+\frac{1}{2} \mathrm{Cl}_{2}\left(2 \arcsin \left(\frac{\mu}{1+\mu}\right)\right)+\int_{0}^{\frac{\mu}{1+\mu}} \frac{\arcsin (x)}{1-x} d x .
\end{gathered}
$$

Оставшийся в (17) интеграл проинтегрируем по частям, а затем перейдем к тригонометрическим функциям с помощью замены переменных $x=\cos (t)$

$$
\begin{aligned}
\int_{0}^{\frac{\mu}{1+\mu}} \frac{\arcsin (x)}{1-x} d x= & \ln (1+\mu) \arcsin \left(\frac{\mu}{1+\mu}\right)+\int_{0}^{\frac{\mu}{1+\mu}} \frac{\ln (1-x)}{\sqrt{1-x^{2}}} d x= \\
& \ln \left(\frac{1+\mu}{2}\right) \arcsin \left(\frac{\mu}{1+\mu}\right)+2 \int_{\arccos \left(\frac{\mu}{1+\mu}\right)}^{\pi / 2} \ln \left(2 \sin \left(\frac{t}{2}\right)\right) d t .
\end{aligned}
$$

Исходя из определения интеграла Клаузена, имеем

$$
\int_{\arccos \left(\frac{\mu}{1+\mu}\right)}^{\pi / 2} \ln \left(2 \sin \left(\frac{t}{2}\right)\right) d t=\mathrm{Cl}_{2}\left(\arccos \left(\frac{\mu}{1+\mu}\right)\right)-\mathrm{Cl}_{2}\left(\frac{\pi}{2}\right),
$$

поэтому после подстановки (18) в (17) приходим к следующему выражению:

$$
I_{2}(1+\mu,-\mu)=\ln (\mu) \arcsin \left(\frac{\mu}{1+\mu}\right)+2 \mathrm{Cl}_{2}\left(\arccos \left(\frac{\mu}{1+\mu}\right)\right)+\frac{1}{2} \mathrm{Cl}_{2}\left(2 \arcsin \left(\frac{\mu}{1+\mu}\right)\right) .
$$

При $\mu=1$ получаем $I_{2}(2,-1)=\frac{5}{2} \mathrm{Cl}_{2}(\pi / 3)$. Это основной результат работы [21], из которого следует, что энтропия равна $S_{t}=5 / \pi \mathrm{Cl}_{2}(\pi / 3)$.

Для завершенности изложения желательно вычислить $I_{2}(1+\mu,-\mu)$ во всей области допустимых значений параметра $\mu$. В ходе проведения расчетов нам потребуются аналоги формул (17) и (18) на промежутке $-1 / 2 \leqslant \mu<0$. Необходимые преобразования сводятся к аккуратному учету знаков в вышеупомянутых выражениях и позволяют представить окончательный результат в виде

$$
\begin{aligned}
I_{2}(1+\mu,-\mu)= & -\ln (|\mu|) \arcsin \left(\frac{|\mu|}{1+\mu}\right)+2 \mathrm{Cl}_{2}\left(\pi-\arccos \left(\frac{|\mu|}{1+\mu}\right)\right)- \\
& \frac{1}{2} \mathrm{Cl}_{2}\left(2 \arcsin \left(\frac{|\mu|}{1+\mu}\right)\right), \quad\left(-\frac{1}{2} \leqslant \mu<0\right) .
\end{aligned}
$$

Подстановка в данное выражение значения $\mu=-\frac{1}{2}$ приводит к известному равенству $I_{2}\left(\frac{1}{2}, \frac{1}{2}\right)=\frac{\pi}{2} \ln 2$, которое обсуждалось в разделе 4 .

Оценки сверху находятся так же, как в модели димеров. В нулевом приближении для энтропии на треугольной решетке получаем неравенство $S_{t}<2 \ln (1+\sqrt{2})$. Погрешность данной оценки составляет примерно $9 \%$.

\section{Заключение}

Результаты работы свидетельствуют о том, что в различных решеточных моделях при вычислении ключевых параметров используются весьма близкие методы, 
которые приводят к интегралам от функций, являющихся логарифмами тригонометрических полиномов. Для многих популярных в приложениях видов решеток эти интегралы попадают в семейство $I_{2}(\mu, \nu)$ и могут быть изучены на основе единых принципов. Особый интерес представляет возможность получения простых, но достаточно точных, оценок сверху для всех членов семейства $I_{2}(\mu, \nu)$.

В то же время подход Монтролла не универсален. Для двухпараметрических семейств графов перспективным представляется дальнейшее развитие метода, получившего в работах по модели димеров название EFM (Exact Finite Method). Этот метод, предложенный в пионерской работе [16], позволяет оценить значение $\phi$ исходя из набора решений однопараметрических задач. С его помощью в $[8,22]$ была с высокой точностью проверена гипотеза о равенстве значений $\phi$ на треугольной решетке с периодическими граничными условиями и со свободной границей.

\section{Список литературы}

[1] Wu F.Y. Dimers on two-dimensional lattices // International Journal of Modern Physics B. 2006. Vol. 20, № 32. Pp. 5357-5371.

[2] Montroll E.W. Lattice statistics // Applied Combinatorial Mathematics / Ed. by E.F. Beckenbach. New York: John Wiley and Sons, 1964. Pp. 96-143.

[3] Прудников А.П., Брычков Ю.А., Маричев О.И. Интегралы и ряды. Элементарные функции. М.: Наука, ФизМатЛит, 1981. 800 с.

[4] Grensing D., Carlsen I., Zapp H.-Chr. Some exact results for the dimer problem on plane lattices with non-standard boundaries // Philosophical Magazine A. 1980. Vol. 41, № 5. Pp. 777-781.

[5] Elser V. Solution of the dimer problem on a hexagonal lattice with boundary // Journal of Physics A: Mathematical and General. 1984. Vol. 17, № 7. Pp. 15091513.

[6] Kasteleyn P.W. The statistics of dimers on a lattice: I. The number of dimer arrangements on a quadratic lattice // Physica. 1961. Vol. 27, № 12. Pp. 12091225 .

[7] Temperley H.N.V., Fisher M.E. Dimer problem in statistical mechanics - an exact result // Philosophical Magazine. 1961. Vol. 6, № 68. Pp. 1061-1063.

[8] Перепечко С.Н. Количество совершенных паросочетаний на треугольных решетках фиксированной ширины // Тезисы докладов международной научной конференции «Дискретная математика, алгебра и их приложения». Минск, 2015. С. 129-130.

[9] Караваев А.М., Перепечко С.Н. Производящие функции в задаче о димерах на прямоугольных сеточных графах // Информационные процессы. 2013. Т. 13, № 4. C. 374-400.

[10] Krupnikov E.D., Kölbig K.S. Some special cases of the generalized hypergeometric function ${ }_{q+1} F_{q} / /$ Journal of Computational and Applied Mathematics. 1997. Vol. 78. Pp. 79-95. 
[11] Schrijver A. Counting 1-factors in regular bipartite graphs // Journal of Combinatorial Theory B. 1998. Vol. 72. Pp. 122-135.

[12] Gordon M., Davison W.T.H. Theory of resonance topology of fully aromatic hydrocarbons. I // Journal of Chemical Physics. 1952. Vol. 20, № 3. Pp. 428435 .

[13] Wannier G.H. Antiferromagnetism. The triangular Ising net // Physical Review. 1950. Vol. 79, №2. Pp. 357-364.

[14] Clausen Th. Über die function $\sin \phi+\frac{1}{2^{2}} \sin 2 \phi+\frac{1}{3^{2}} \sin 3 \phi+$ etc. // Journal für die reine und angewandte Mathematik. 1832. Vol. 8. Pp. 298-300.

[15] Fettis H.E. Problem 6448 // American Mathematical Monthly. 1984. Vol. 91, № 1. P. 59.

[16] Fowler R.H., Rushbrooke G.S. An attempt to extend the statistical theory of perfect solutions // Transactions of the Faraday Society. 1937. Vol. 33. Pp. 12721294.

[17] Izmailian N.Sh., Oganesyan K.B., Wu M.-Ch., Hu Ch.-K. Finite-size corrections and scaling for the triangular lattice dimer model with periodic boundary conditions // Physilal Review E. 2006. Vol. 73. Art. 016128.

[18] Phares A.J., Wunderlich F.J. Thermodynamics and molecular freedom of dimers on plane triangular lattices // Journal of Mathematical Physics. 1986. Vol. 27. Pp. 1099-1109.

[19] Приезжев В.Б. Задача о димерах и теорема Кирхгофа // Успехи физических наук. 1985. Т. 147, № 4. С. 747-765.

[20] Guttmann A.J., Rogers M.D. Spanning tree generating functions and Mahler measures // Journal of Physics A: Mathematical and Theoretical. 2012. Vol. 45. Art. 494001.

[21] Glasser M.L., Wu F.Y. On the entropy of spanning trees on a large triangular lattice // Ramanujan Journal. 2005. Vol. 10. Pp. 205-214.

[22] Перепечко С.Н. Оценка параметра молекулярная свобода в модели димеров методом EFM // Материалы VI международной конференции «Математика, ее приложения и математическое образование». Улан-Удэ, 2017. С. 289-294.

\section{Образец цитирования}

Перепечко С.Н. Простые выражения для оценки параметра молекулярная свобода в задаче о димерах // Вестник ТвГУ. Серия: Прикладная математика. 2018. № 2. C. 27-47. https://doi.org/10.26456/vtpmk194 


\section{Сведения об авторах}

\section{1. Перепечко Сергей Николаевич}

доцент кафедры прикладной математики и кибернетики Петрозаводского государственного университета.

Россия, 185910, Республика Карелия, е. Петрозаводск, пр. Ленина, д. З3, ПетрГУ. E-mail: persn@newmail.ru 


\section{SIMPLE EXPRESSIONS FOR ESTIMATING THE MOLECULAR FREEDOM PARAMETER IN THE DIMER PROBLEM}

Perepechko Sergey Nikolaevich

Associate professor at Applied Mathematics and Cybernetics department, Petrozavodsk State University

Russia, 185910, Republic of Karelia, Petrozavodsk, 33 Lenin str., PetrSU. E-mail: persn@newmail.ru

Received 24.10.2017, revised 05.02.2018.

A method for calculating the molecular freedom parameter in the dimer model for two-parameter families of lattice graphs with periodic boundary conditions is discussed. It is shown that the scheme of calculations for the most known types of lattices can be unified and it allows us to find simple but fairly accurate estimates from above of the model parameter. The possibilities of the proposed approach are illustrated by examples for which only numerical values were previously known. It is noted that the method can also be useful for estimating parameters in other lattice models. We show that the entropy of spanning trees on a triangular lattice is a special case of the formulas derived in this paper.

Keywords: dimer problem, molecular freedom.

\section{Citation}

Perepechko S.N. Simple expressions for estimating the molecular freedom parameter in the dimer problem. Vestnik TvGU. Seriya: Prikladnaya Matematika [Herald of Tver State University. Series: Applied Mathematics], 2018, no. 2, pp. 27-47. (in Russian). https://doi.org/10.26456/vtpmk194

\section{References}

[1] Wu F.Y. Dimers on two-dimensional lattices. International Journal of Modern Physics B, 2006, vol. 20(32), pp. 5357-5371.

[2] Montroll E.W. Lattice statistics. Applied Combinatorial Mathematics. Ed. by E.F. Beckenbach. John Wiley and Sons, New York, 1964, pp. 96-143.

[3] Prudnikov A.P., Brychkov Yu.A., Marichev O.I. Integraly i ryady. Tom 1. Elementarnye functii [Integrals and Series. Volume 1. Elementary functions]. Nauka, FizMatLit Publ., Moscow, 1981. 800 p. (in Russian)

[4] Grensing D., Carlsen I., Zapp H.-Chr. Some exact results for the dimer problem on plane lattices with non-standard boundaries. Philosophical Magazine A, 1980, vol. 41(5), pp. $777-781$. 
[5] Elser V. Solution of the dimer problem on a hexagonal lattice with boundary. Journal of Physics A: Mathematical and General, 1984, vol. 17(7), pp. 1509-1513.

[6] Kasteleyn P.W. The statistics of dimers on a lattice: I. The number of dimer arrangements on a quadratic lattice. Physica, 1961, vol. 27(12), pp. 1209-1225.

[7] Temperley H.N.V., Fisher M.E. Dimer problem in statistical mechanics - an exact result. Philosophical Magazine, 1961, vol. 6(68), pp. 1061-1063.

[8] Perepechko S.N. Number of perfect matchings on triangular lattices of fixed width. Proceedings of the conference "Discrete Mathematics, Algebra and Their Applications"(DIMA-2015), Minsk, 2015, pp. 129-130.

[9] Karavaev A.M., Perepechko S.N. Generating functions for dimer problem on rectangular lattices. Informatsionnye protsessy [Information Processes], 2013, vol. 13(4), pp. 374-400.

[10] Krupnikov E.D., Kölbig K.S. Some special cases of the generalized hypergeometric function ${ }_{q+1} F_{q}$. Journal of Computational and Applied Mathematics, 1997, vol. 78, pp. 79-95.

[11] Schrijver A. Counting 1-factors in regular bipartite graphs. Journal of Combinatorial Theory B, 1998, vol. 72, pp. 122-135.

[12] Gordon M., Davison W.T.H. Theory of resonance topology of fully aromatic hydrocarbons. I. Journal of Chemical Physics, 1952, vol. 20(3), pp. 428-435.

[13] Wannier G.H. Antiferromagnetism. The triangular Ising net. Physical Review, 1950, vol. 79(2), pp. 357-364.

[14] Clausen Th. Über die function $\sin \phi+\frac{1}{2^{2}} \sin 2 \phi+\frac{1}{3^{2}} \sin 3 \phi+$ etc. Journal für die reine und angewandte Mathematik, 1832, vol. 8, pp. 298-300.

[15] Fettis H.E. Problem 6448. American Mathematical Monthly, 1984, vol. 91(1), p. 59.

[16] Fowler R.H., Rushbrooke G.S. An attempt to extend the statistical theory of perfect solutions. Transactions of the Faraday Society, 1937, vol. 33, pp. 12721294.

[17] Izmailian N.Sh., Oganesyan K.B., Wu M.-Ch., Hu Ch.-K. Finite-size corrections and scaling for the triangular lattice dimer model with periodic boundary conditions. Physilal Review E, 2006, vol. 73, art. 016128.

[18] Phares A.J., Wunderlich F.J. Thermodynamics and molecular freedom of dimers on plane triangular lattices. Journal of Mathematical Physics, 1986, vol. 27, pp. 1099-1109.

[19] Priezzhev V.B. The dimer problem and the Kirchhoff theorem. Soviet Physics Uspekhi, 1985, vol. 28(12), pp. 1125-1135.

[20] Guttmann A.J., Rogers M.D. Spanning tree generating functions and Mahler measures. Journal of Physics A: Mathematical and Theoretical, 2012, vol. 45, art. 494001. 
[21] Glasser M.L., Wu F.Y. On the entropy of spanning trees on a large triangular lattice. Ramanujan Journal, 2005, vol. 10, pp. 205-214.

[22] Perepechko S.N. Estimation of molecular freedom in the dimer model by the EFM method. Proceedings of the VI international conference "Mathematics, its applications and mathematical education"(MAME-2017), Ulan-Ude, 2017, pp. 289-294. 